\title{
Effect of bisphosphonates on the mandibular bone and gingival epithelium of rats without tooth extraction
}

\author{
FRANCESCO SAVERIO DE PONTE ${ }^{1}$, LUCIANO CATALFAMO ${ }^{1}$, GREGORIO MICALI ${ }^{1}$, MICHELE RUNCI ${ }^{1}$, \\ GIUSEPPINA CUTRONEO ${ }^{2}$, GIOVANNA VERMIGLIO ${ }^{2}$, ANTONIO CENTOFANTI ${ }^{2}$ and GIUSEPPINA RIZZO $^{2}$ \\ ${ }^{1}$ Unit of Maxillo-Facial Surgery, Department of Odonostomatology; \\ ${ }^{2}$ Department of Biomedical Sciences and Morpho-Functional Images, University of Messina, I-98125 Messina, Italy
}

Received December 22, 2014; Accepted November 30, 2015

DOI: $10.3892 /$ etm.2016.3168

\begin{abstract}
Osteonecrosis of the jaw (ONJ) is an adverse effect of bisphosphonate treatment that has become the subject of increasing investigations, in particular due to its poorly understood pathogenesis. Several experimental studies on animal models have been conducted; however, the majority of these replicate human $\mathrm{ONJ}$ following tooth extraction, and describe alterations in the bone and gingival epithelium when necrosis is manifested. The aim of the present study was to analyze the rat mandibular bone and gingival epithelium during 45 days of zoledronate treatment (which is a bisphosphonate agent), without tooth extraction. Intraperitoneal injections of zoledronate acid $(0.1 \mathrm{mg} / \mathrm{kg})$ were performed three times a week in normal male Wistar rats $(n=20)$, while a control group of rats $(n=20)$ was treated with saline solution for 45 days. After 7 , 15,30 and 45 days of drug treatment, all rats were sacrificed and hematoxilin and eosin staining, immunofluorescence and scanning electron microscopy analyses were performed. The results of the analyses after 7 and 15 days of treatment were similar in the treatment and control group. After 30 and 45 days of treatment, structural alterations were observed in the bone. No structural alterations to the gingival epithelium were observed. Based on these results, it was hypothesized that low doses of zoledronate act directly on the bone tissues to induce morphological alterations from bone to necrotic tissue following surgical procedures, although no cytotoxic effects were detected in the gingival epithelium.
\end{abstract}

Correspondence to: Professor Giuseppina Cutroneo, Department of Biomedical Sciences and Morpho-Functional Images, University of Messina, 1 Via Consolare Valeria, I-98125 Messina, Italy

E-mail: gcutroneo@unime.it

Abbreviations: BONJ, bisphosphonate-related osteonecrosis of the jaw; ONJ, osteonecrosis of the jaw; PDL, periodontal ligament

Key words: bisphosphonates, mandibular bone, gingival epithelium, osteblasts, osteoclasts

\section{Introduction}

Bisphosphonates (BPs) are synthetic analogues of inorganic pyrophosphates, which act as potent inhibitors of osteoclast-mediated bone resorption $(1,2)$. This type of drug is currently used in various pathological conditions, including postmenopausal osteoporosis, hypercalcemia associated with malignancy, lytic bone metastasis and other metabolic bone diseases $(3,4)$. The two most potent and widely used nitrogen-containing BPs are zoledronate and alendronate, which inhibit the intracellular mevalonate pathway $(5,6)$. The target protein of the nitrogen-containing BPs is considered to be farnesyl diphosphate synthase, an important regulatory enzyme for isoprenoid lipid production (6).

Certain isoprenoid lipids, including farnesyl pyrophosphate and geranyl-geranyl pyrophosphosphate, serve an important role in the prenylation and activation of small GTPases, such as Ras, rhodopsin, Rac, Rab and cell division cycle 42 (7). In turn, the small GTPases function as signaling proteins involved in the regulation of osteoclast morphology, cytoskeleton arrangement, membrane ruffling, trafficking and cell survival (8-11). Currently, the resulting adverse effects from BP treatment are an important issue. Although BPs are beneficial for several pathological conditions of the bone, BP-related osteonecrosis of the jaw (BONJ) has been reported (12). BONJ has been predominantly observed in patients who underwent mechanical trauma, such as tooth extraction, or were affected by periodontal disease, as well as patients receiving corticosteroid treatment $(1,13,14)$. Despite the importance of the problem and numerous studies investigating BONJ since $2004(1,13,14)$, BONJ etiology and pathophysiology remain largely unknown. Several hypotheses have been proposed, including BP toxicity to oral epithelium, altered wound healing following tooth extraction, high turnover of the mandible and maxilla, oral biofilm formation, infection and inflammation, suppression of angiogenesis and bone turnover, and osteoblast death (15-19).

Our previous studies $(20,21)$ conducted on epithelial and bone tissue samples from patients with BONJ demonstrated the presence of structural alteration of the bone; furthermore, a decrease or absence of adhesion proteins, sarcoglycans and integrins, was observed in the gingival epithelium of patients with BONJ. Therefore, it was hypothesized that two different pathogenic processes lead to BONJ: An indirect process, from 
the oral mucosa to the bone, and a direct process, from the bone to the mucosa $(20,21)$.

ONJ is a complex disease that involves the interaction of multiple tissues and cell types in response to local wound healing; it is therefore difficult to replicate the disease conditions in vitro. Various animal models replicating the clinical, radiographic and histologic features of ONJ have been developed in rats, mice and mini pigs (22-24). The majority of these animal models underwent tooth extraction following prolonged periods with high-dose BP treatment, suggesting that BPs alter bone healing and lead to bone exposure. However, a significant fraction of ONJ diseases occurs in the absence of tooth extraction.

Therefore, the present study used a rat model to observe the bone and gingiva during 45 days of zoledronate treatment, without tooth extraction. The aim of the present investigation was to analyze the changes that occur in the bone and in the gingiva during treatment with BPs in order to determine the mechanisms underlying the progression of bone osteonecrosis. Furthermore, based on the two previous hypotheses the study also aimed to determine which process, direct or indirect, leads to BONJ.

\section{Materials and methods}

Animals. A total of 40 male Wistar rats (age, 7 weeks) were purchased from Janvier Labs (Saint Berhevin, France). The rats were housed in individual cages with bedding, and standard rat food and tap water were available ad libitum for the duration of the experimental period, unless otherwise noted. The rats were maintained under a 12-h light/dark cycle at a constant temperature of $22.0 \pm 0.6^{\circ} \mathrm{C}$. The rat handling procedures were conducted in accordance with the European Communities Council Directive of the 24th November 1986 (86/609/EEC). All experimental protocols were approved by the Committee for Animal Care and Use at the University of Messina (Messina, Italy). A total of 20 rats were treated with intraperitoneal injections of $0.1 \mathrm{mg} / \mathrm{kg}$ zoledronate (Novartis Pharmaceuticals Corporation East Hanover, NJ, USA) three times a week, and an additional 20 rats were injected with saline as a control. Doses and time schedules of drug administration were designed according to previous studies $(25,26)$. After 7, 15, 30 and 45 days of treatment, 5 rats from each group were sacrificed by paraformaldehyde (Sigma-Aldrich, St. Louis, MO, USA) injection into the left ventricle of the heart, and their mandibles were harvested and divided in two parts. One part was used for scanning electron microscopy (SEM) and the second part was used for histological and immunofluorescence analyses. In addition, gingival epithelia biopsies were obtained and used for histological and immunofluorescence analyses.

SEM. The tissue specimens were fixed with $2 \%$ glutaraldehyde (Santa Cruz Biotechnology, Inc., Dallas, TX, USA) in $0.1 \mathrm{M}$ phosphate buffer (Sigma-Aldrich) at $\mathrm{pH} 7.4$ at room temperature. The specimens were dehydrated through a gradual increase in the concentration of ethanol and amyl acetate (1st solution: $25 \%$ ethanol, $25 \%$ amyl acetate, $50 \%$ distilled water; 2 nd solution: $35 \%$ ethanol, $35 \%$ amyl acetate, $30 \%$ distilled water; 3rd solution: $40 \%$ ethanol, $40 \%$ amyl acetate, $20 \%$ distilled water; 4th solution: $45 \%$ ethanol, $45 \%$ amyl acetate, $10 \%$ distilled water and; 5 th solution: $50 \%$ ethanol and 50\% amyl acetate; Merck Millipore, Darmstadt, Germany). Subsequently, the tissue specimens were dried at critical-point in a Leica EM CPD030 Critical Point Dryer (Leica Microsystems GmbH, Wetzlar, Germany) using liquid $\mathrm{CO}_{2}$. The fractured surface of the mandible was mounted on stub supports (Tousimis, Rockville, MD, USA) and platinum coated with a Plasma Sciences CrC-100 Turbo-Pumped sputtering system (Electron Microscopy Sciences, Hatfield, PA, USA), and observed using a Phenom G2 Pro scanning electron microscope (Phenom-World B.V., Eindhoven, The Netherlands).

Histological analysis. Following perfusion, the tissue specimens were post-fixed with $2 \%$ glutaraldehyde and $12.5 \%$ formaldehyde (Sigma-Aldrich), and buffered in 0.1 M sodium cacodylate ( $\mathrm{pH} 7.4$; Sigma-Aldrich) at room temperature for $4 \mathrm{~h}$. Following rinses in $13 \mathrm{M}$ phosphate buffer $(\mathrm{pH} 7.3)$, the tissue specimens were decalcified in $4.13 \%$ ethylenediaminetetraacetic acid ( $\mathrm{pH} 7.2$; Hach Company, Loveland, CO, USA) for 30 days, dehydrated in ethanol and embedded in paraffin. Tissue sections $(5 \mathrm{~mm})$ were obtained using the Leica RM2255 microtome (Leica Microsystems $\mathrm{GmbH}$ ) and stained with hematoxylin and eosin (H\&E; Abbey Color, Philadelphia, PA, USA) for 15 and $5 \mathrm{~min}$, respectively, at room temperature.

Immunofluorescence. Non-colored sections of bone and gingiva $(10 \mathrm{~mm})$, prepared during the histological analysis were deparaffinized twice in xylene (5 min each; Sigma-Aldrich), hydrated twice in $100 \%$ ethanol (3 min each), $95 \%$ ethanol (1 min), 90\% ethanol (1 min) and 80\% ethanol (1 min), and rinsed in distilled water. Pre-heating was conducted in a MW 200 steamer (De'Longhi Appliances S.r.1, Treviso, Italy) with a staining dish containing sodium citrate buffer (Sigma-Aldrich) at $95-100^{\circ} \mathrm{C}$.

To block non-specific sites and to make the membranes permeable, the mandible and gingiva tissue sections were pre-incubated with $1 \%$ bovine serum albumin and $0.3 \%$ Triton X-100 (both Sigma-Aldrich) in phosphate-buffered saline at room temperature for $15 \mathrm{~min}$. Next, the tissue sections were incubated with primary antibodies at room temperature for $2 \mathrm{~h}$. For the mandible tissue sections, the following primary antibodies were used: Rabbit polyclonal anti-receptor activator of nuclear factor- $\kappa$ B (RANK; 1:150; sc-9072; Santa Cruz Biotechnology, Inc.) to detect osteoclasts, and rabbit polyclonal anti-osteocalcin (1:200; sc-30045; Santa Cruz Biotechnology, Inc.) to detect osteoblasts. For gingival epithelia tissue sections, goat polyclonal anti- $\varepsilon$-sarcoglycan $(1: 100$; Santa Cruz Biotechnology, Inc.) was used. Incubation with anti-RANK and anti-e-sarcoglycan primary antibodies was followed by incubation with Texas Red-conjugated anti-goat (305-075-047) and Rhodamine Red-conjugated anti-rabbit (711-295-152) IgG (heavy\&light chains), respectively (1:100; Jackson ImmunoResearch Laboratories, Inc., West Grove, PA, USA), at room temperature for $1 \mathrm{~h}$. Incubation with anti-osteocalcin antibody was followed by incubation with fluorescein isothiocyanate-conjugated anti-rabbit fluorochrome (1:100; 111-095-046; Jackson ImmunoResearch Laboratories, Inc.) at room temperature for $1 \mathrm{~h}$. The tissue samples were observed 

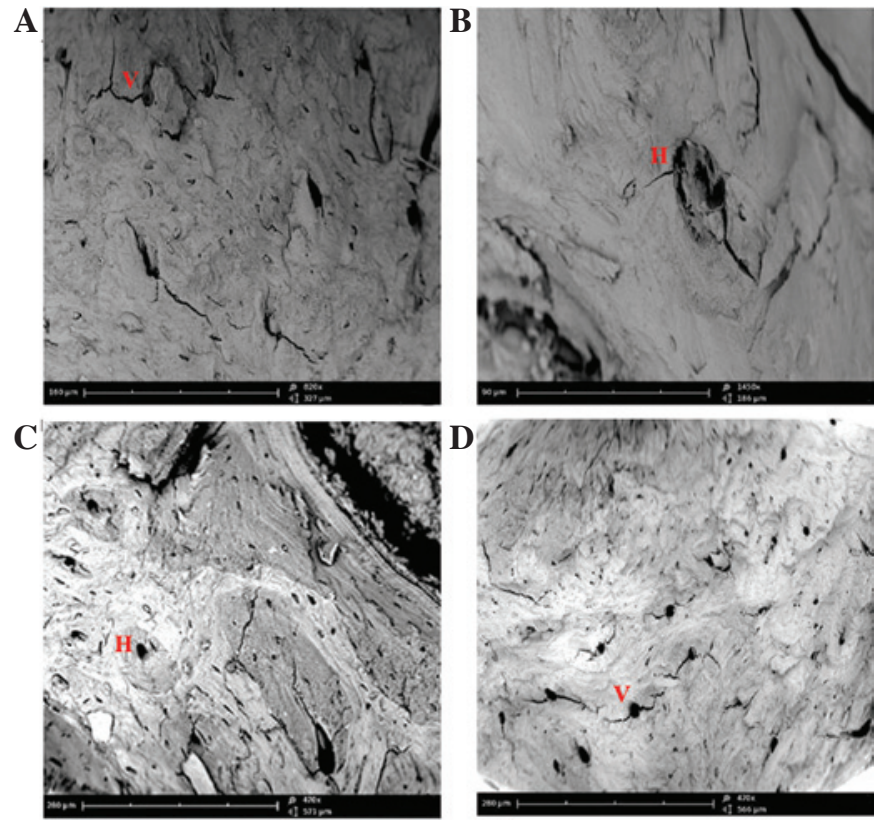

Figure 1. Scanning electron microscopy of the rat mandibular bone after 7-15 and 30-45 days of zoledronate treatment $(0.1 \mathrm{mg} / \mathrm{kg})$. In the $7-15$ days group, (A) full osteocytic lacunae and full Volkman's canals were observed, as well as (B) Havers' canals. In the 30-45 days group, (C) empty osteocytic lacunae and empty Havers' canals were observed, as well as (D) Volkman's canals. V, Volkman's systems; H, Haversian systems.

with a Zeiss LSM 510 confocal microscope (Zeiss AG, Oberkochen, Germany) equipped with an Argon laser (458 nm and $488 \mathrm{~nm} \lambda$ ) and two HeNe lasers (543 $\mathrm{nm}$ and $633 \mathrm{~nm} \lambda$ ). All images were digitized at a resolution of 8 bits into an array of $2,048 \times 2,048$ pixels. Optical sections of the fluorescent tissue specimens were obtained at $488 \mathrm{~nm} \lambda$, and 62/sec scanning shipped with $\geq 8$ repetitions on average. The detection pinhole was set for optimal resolution. Contrast and brightness were established by examining the most brightly labelled pixels and selecting settings that allowed clear visualization of structural details while keeping the highest pixel intensities 200 . Digital images were cropped and figure montages prepared using Adobe Photoshop 7.0 (Adobe Systems, Inc., San Jose, CA, USA).

\section{Results}

BP treatment. The bone and gingiva tissue samples from all rats were observed by H\&E staining, SEM and immunofluorescence. No differences were observed in the bone and gingiva tissue samples after 7 and 15 days of zoledronate $(0.1 \mathrm{mg} / \mathrm{kg})$ treatment, as compared with the control group. In addition, the results observed after 7 days were similar to those observed after 15 days of treatment. In the bone and gingiva tissue samples, several changes were observed after 30 and 45 days of treatment, as compared with the bone and gingiva tissue samples treated for 7 or 15 days, and the control group. The results observed at 30 and 45 days of treatment were similar. Based on these results, the tissue specimens were described and compared in two groups, including the 7-15 and 30-45 groups, according to the number of days after zoledronate treatment. No cases of necrosis or bone exposure were observed.
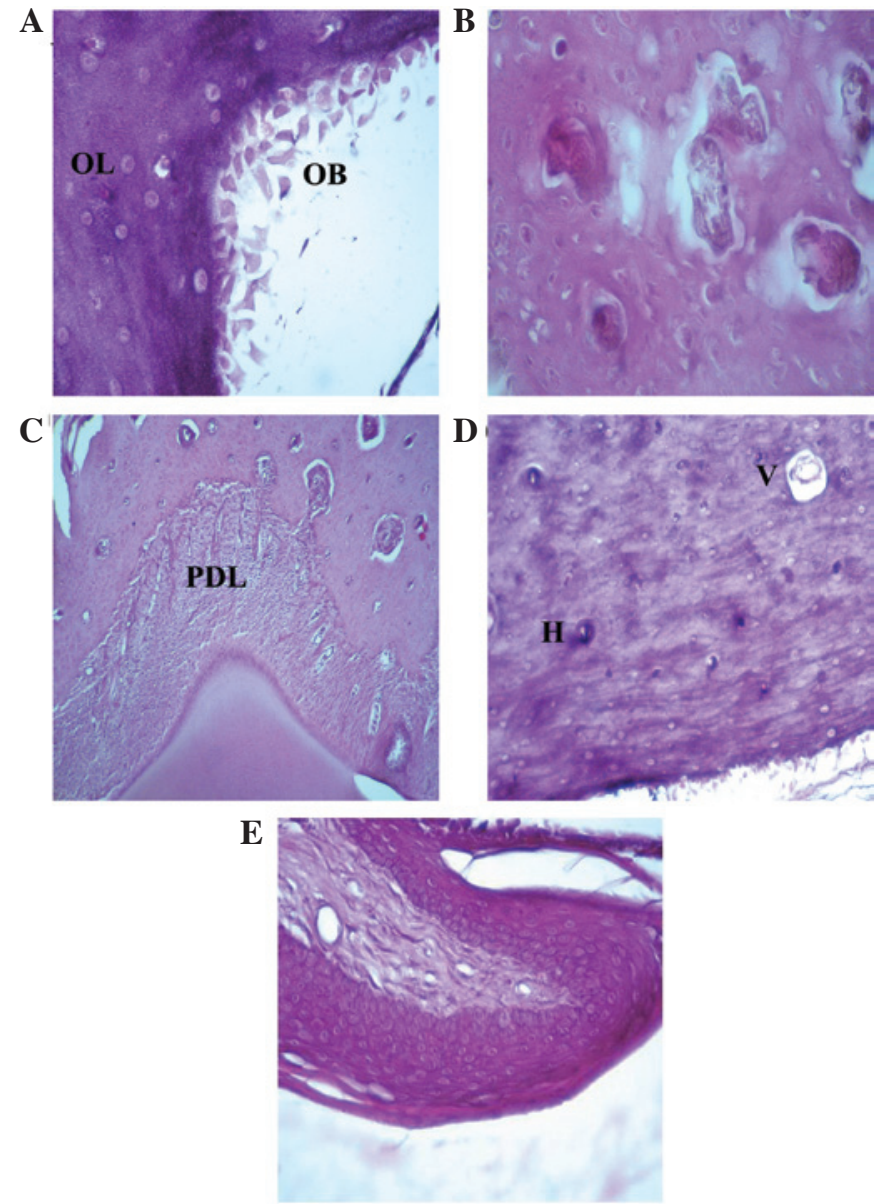

Figure 2. Hematoxilin and eosin staining of the rat mandibular bone and gingival epithelium after $7-15$ days of zoledronate treatment $(0.1 \mathrm{mg} / \mathrm{kg})$ (A) Osteoblasts at the rim side of the cortical bone and full osteocytic lacunae were observed (magnification, $\mathrm{x} 40$ ). Hematoxylin and eosin staining also demonstrated the presence of (B) full Howship's lacunae (magnification, $\mathrm{x} 40$ ), (C) normal periodontal ligament between the tooth root and the bone (magnification, x10) and (D) full Volkman's and Haversian systems (magnification, x20). (E) Normal gingival epithelium structure (magnification, x20). OB, osteoblast; OL, osteocytic lacunae; PDL, periodontal ligament; V, Volkman's systems; H, Haversian systems.

SEM. The images of the 7-15 group (Fig. 1) highlighted the presence of full osteocytic lacunae, Haversian systems and Volkmann canals in the cortical and trabecular alveolar bone regions. Conversely, images of the 30-45 (Fig. 1) group showed numerous empty osteocytic lacunae, which were particularly abundant in the cortical bone. Empty Haversian systems and Volkmann canals were also observed.

$H \& E$ staining. The mandibular bones after 7-15 days of zoledronate treatment (Fig. 2) exhibited similar characteristics to healthy bones, as evidenced by full osteocytic lacunae, presence of osteoblasts at the mineralization sides, full Howship's lacunae with osteoclastic rims, normal Haversian' systems, and Volkmann's canals. The normal structure of surrounding periodontal ligament and gingival mucosa were also observed.

Mandibular bones after 30-45 days of zoledronate treatment (Fig. 3) exhibited structural modifications as evidenced by empty osteocytic lacunae, which were particularly abundant in the cortical bone surrounding the alveolar socket. The presence of empty Howship's lacunae, Haversian systems 

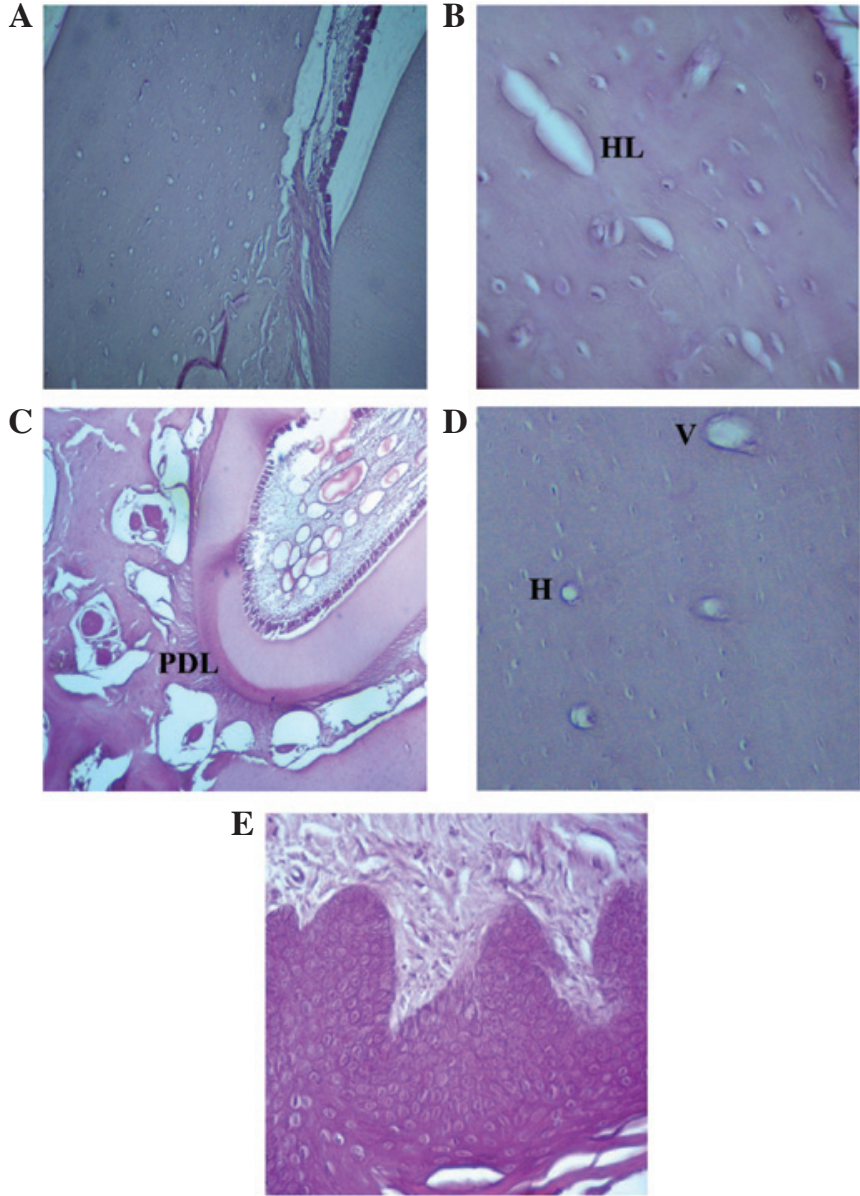

Figure 3. Hematoxilin and eosin staining of the rat mandibular bone and gingival epithelium following $30-45$ days of zoledronate treatment $(0.1 \mathrm{mg} / \mathrm{kg})$. (A) Osteoblasts at the rim side of the cortical bone and empty osteocytic lacunae (magnification, x10), as well as (B) Howship's lacunae (magnification, $\mathrm{x} 40$ ) were observed. Hematoxylin and eosin staining also demonstrated the presence of $(\mathrm{C})$ a detachment of the periodontal ligament from the bone (magnification, x20), as well as (D) empty Volkman's and Haversian systems (magnification, x20). (E) Normal gingival epithelium structure (magnification, x20). HL, Howship's lacunae; PDL, periodontal ligament; V, Volkman's systems; H, Haversian systems.

and Volkmann canals was also noted. No osteoblastic rims were observed in a wide area of bone surrounding the tooth root region, as evidenced by the absence of osteoblasts in the mineralized side. Occasionally, the surrounding tissue samples exhibited altered structure, as evidenced by abnormal gaps between the periodontal ligament and bone; however, the gingival epithelium and connective tissue showed normal organization. Regions of healthy bone were also observed in these samples.

Immunofluorescence. Immunofluorescence analysis of the 7-15 group specimens (Fig. 4A-D) showed the presence of osteoclasts and osteoblasts, as evidenced by the RANK (red) and osteocalcin (green) markers, respectively. Osteoclasts were detected in Howship's lacunae. Conversely, bone specimens in rats treated with zoledronate for 30-45 days (Fig. 4E-H) exhibited a marked reduction in osteoblast fluorescence, specifically in the cortical bone surrounding the alveolar socket. Furthermore, the images showed a weak osteoclast fluorescence pattern, and absence of fluorescence
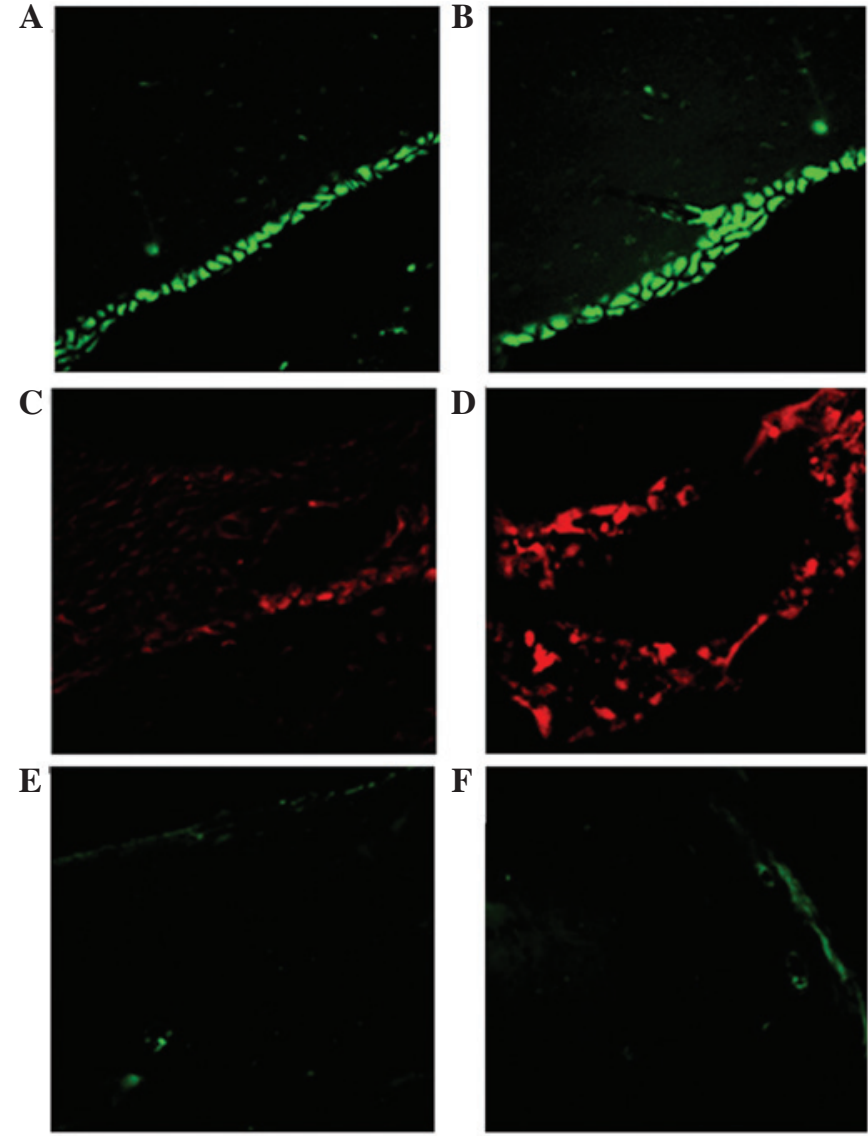

$\mathbf{G}$

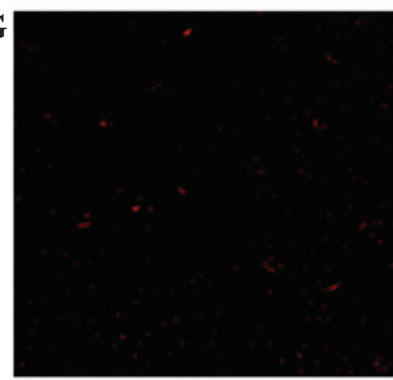

H

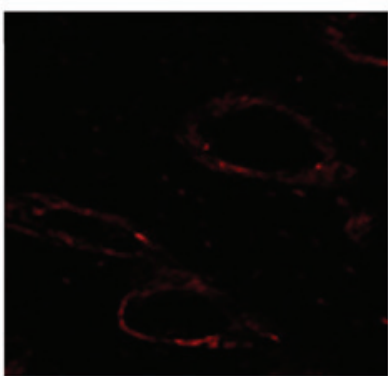

I
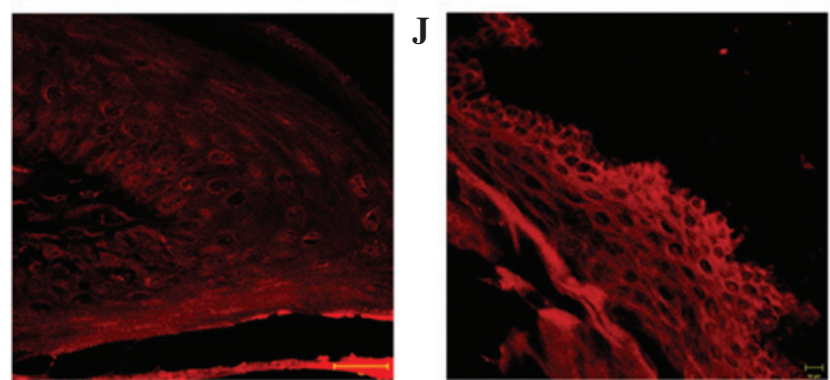

Figure 4. Immunofluorescence staining of the rat mandibular bone and gingival epithelium following 7-15 and 30-45 days of zoledronate treatment $(0.1 \mathrm{mg} / \mathrm{kg})$. In the $7-15$ day group, (A and B) osteoblasts were present at the mineralization side, as determined by osteocalcin labeling (green), as well as (C and D) the presence of osteoclasts, labeled by RANK (red), in the Howship's lacunae. In the 30-45 days group a near total absence of the tested proteins, (E and F) osteocalcin and (G and $\mathrm{H}$ ) RANK was observed. (E) In all layers of the gingival epithelium a uniform $\alpha$-sarcoglycan pattern of fluorescence (red) was observed in the (I) 7-15 and (J) 30-45 day groups. Magnifications are $\mathrm{x} 40$ for A-H, x63 for I and x20 for J. RANK, receptor activator of nuclear factor- $\kappa \mathrm{B}$.

in several Howship's lacunae. Immunofluorescence analysis of the gingival epithelium demonstrated the presence of a uniform distribution pattern of sarcoglycans (red channel) 
along the epithelial layers in both the 7-15 and 30-45 groups (Fig. 4I and J, respectively).

\section{Discussion}

BP therapy is currently used to contrast several bone pathological conditions, including multiple myeloma, metastatic disease of the skeleton, Paget's disease of the bone and osteoporosis $(3,4)$. This type of drug inhibits osteoclast bone resorption, thereby inhibiting loss of bone mass. It is well established that a recognized side effect of BP is BONJ (12). The etiology and pathogenesis of BONJ remain unclear, although several hypotheses have been proposed. It was initially proposed that a hypoxic/ischemic signaling pathway leads to BONJ, due to the anti-angiogenic properties of the drugs $(17,18)$. Currently, the most accredited hypothesis suggests that the accumulation of BPs in the bone inhibits osteoclast activity and their ability to maintain the bone integrity (8-11). BPs also inhibit the regenerative mechanisms underlying periodontal disease, tooth extraction and invasive surgical treatment in the alveolar bone (13). BONJ has been predominantly observed in patients who underwent surgical treatment, such as tooth extractions or implant placement, or following traumatic events $(13,14)$. However, the lesions may also occur spontaneously with no history of surgical procedures, trauma or radiation therapy.

Our previous studies, conducted on intrasurgical perilesional bone and gingiva tissue biopsies from patients with BONJ, demonstrated the presence of bone structural modifications with empty lacunae, absence of matrix and presence of unorganized fibrillar structures $(20,21)$. In the gingival epithelium a decrease or absence of sarcoglycan and integrin transmembrane proteins was observed (20), proteins which have a role in cell-cell and cell-matrix adhesion (27-29). In addition, an increase in vascular endothelial growth factor expression was observed (21). The observation of structural alterations in the gingival epithelium and bone suggested that BPs may act via two processes: A direct process or an indirect process (21). In the direct process, BPs may act directly on the bone to induce matrix necrosis, particularly in the maxilla and mandibular bone due to their high levels of bone remodeling from the constant stress of the masticatory forces (21). In the indirect process, drugs may induce epithelial modification and absence of cell-cell and cell-matrix adhesion, which allows bacterial transit to the bone in the oral region causing osteomyelitis (21).

The limitation of the aforementioned studies, as well as of investigations on a human model, is the difficulty of successful bone and gingiva tissue sample analysis prior to necrosis. Therefore, it has yet to be established whether the observed damage depends on necrotic or surgical events, specifically in the case of the gingiva. The present study used a rat experimental model in order to circumvent these problems. Previous studies using rat models have already been conducted $(30,31)$, although the majority of these replicate human clinical and histological features of BONJ by investigating the mandibular bone or gingival epithelium following tooth extraction, when bone necrosis has already begun.

A previous report described the bone and gingival epithelium of rats prior to tooth extraction, demonstrating the presence of structural alteration of the bone, as evidenced by empty osteocytic lacunae and inflammation in the gingival mucosa (22). However, the study used histological techniques alone, and gingival inflammation was not fully characterized.

In the present study, the rat mandibular bone and gingiva were observed after 7, 15, 30 and 45 days of zoledronate treatment without tooth extraction. The results, obtained by SEM, hematoxylin and eosin staining, and immunofluorescence demonstrated that the mandibular bone at 7 and 15 days of treatment exhibited similar features to those of healthy bone. Only after 30 days of treatment it was possible to observe structural alteration in large surface areas of the bone, as evidenced by empty osteocytic lacunae, and a marked reduction in the number of osteoblasts and osteoclasts. According to a previous investigation, these morphological features depend on molecular mechanisms underlying BP inhibition of the mevalonate signaling pathway; BPs act by preventing the prenylation and activation of small GTPases that are essential for the bone-resorbing activity and survival of osteoclasts (32). Furthermore, another study suggested that BPs may also have toxic effects on osteoblasts (19). It is known that osteoblasts are important to osteoclast differentiation and activation through the release of osteoclast-activating factors. BPs may induce a reduction in osteoclasts and osteoblasts; in turn, reduction of osteoblasts may inhibit bone-resorbing activity, and the survival of osteoclasts may induce osteonecrosis.

The results of the present study also demonstrated the presence of empty Haversian and Volkman's canals. These observations may be explained by the inhibitory effect of BPs on angiogenesis $(17,18)$, which is of particular interest since ONJ lesions may result from ischemic changes to the tissues. Conversely, another study suggested that BPs exerted a dose-dependent effect on vascularization, and no anti-angiogenic effect was observed with clinical dosing (33). Although several hypotheses have been proposed, the anti-angiogenic mechanisms underlying the effects of BPs remain to be elucidated, and further investigations are required to identify specific markers of angiogenesis.

In two rats, detachment of the periodontal ligament from the bone was observed. It has been demonstrated that nitrogen-containing BPs, upon release from the jawbones in which they previously accumulated, may have a cytotoxic effect on various cells located around the jawbones (34). Furthermore, a decrease in osteoclast activity was demonstrated to influence the differentiation of periodontal ligament cells $(35,36)$. However, the data of the present study was not statistically significant and, for this reason, the observations are not attributable to the effect of BPs.

An important finding of the present report was the absence of gingival epithelium lesions as evidenced by H\&E staining, which demonstrated the presence of a normal epithelial structure. Furthermore, immunofluorescence showed that $\alpha$-sarcoglycan expression was present in all tissue specimens. The sarcoglycan family includes six transmembrane glycoproteins that have a role in cell-cell and cell-matrix adhesion (37-39). Therefore, the presence of $\alpha$-sarcoglycan indicated the absence of structural and functional alteration in the gingival epithelium in the 7-15 and 30-45 day treatment groups. The presence of structural modifications of the bone but no gingival lesions further support the hypothesis of a direct pathogenic event leading to BONJ. Furthermore, the 
present study used an animal model to understand which are the pathogenic events that occur in a short time frame of BP treatment without spontaneous or surgically-induced osteonecrosis. It is possible to speculate that this animal model may replicate human conditions (BP treatment in the absence of spontaneous or surgically-induced osteonecrosis). On this basis, it is possible to consider the mandibular bones of patients treated with BPs as bones which have lost healthy features. This state at the early stage of BONJ was defined as stage 0 according to the modified AAOMS criteria. A lost feature may be the regenerative ability required following tooth extraction and invasive surgical treatment of the alveolar bone, allowing bacterial infections and necrotic bone exposure. This condition may be defined as the late stage of BONJ, corresponding to stages 2 and 3 according to the modified AAOMS criteria (40).

In conclusion, the present study aimed to analyze the rat mandibular bone and gingival epithelium during 45 days of zoledronate treatment without tooth extraction, in order to determine the mechanisms underlying the progression of bone osteonecrosis. The results of the present study demonstrated that, after 30 and 45 days with low dose zoledronate treatment, the mandibular bone underwent morphological changes that may predispose the bone to necrosis when trauma is applied. Further studies should focus on animal models to further investigate the role of BPs in the development of BONJ, in order to develop a therapeutic strategy.

\section{References}

1. Merigo E, Manfredi M, Meleti M, Guidotti R, Ripasarti A Zanzucchi E, D'Aleo P, Corradi D, Corcione L, Sesenna E, et al: Bone necrosis of the jaws associated with bisphosphonate treatment: A report of twenty-nine cases. Acta Biomed 77: 109-117, 2006.

2. Gutta R and Louis PJ: Bisphosphonates and osteonecrosis of the jaws: Science and rationale. Oral Surg Oral Med Oral Pathol Oral Radiol Endod 104: 186-193, 2007.

3. Migliorati CA, Schubert MM, Peterson DE and Seneda LM: Bisphosphonate-associated osteonecrosis of mandibular and maxillary bone: An emerging oral complication of supportive cancer therapy. Cancer 104: 83-93, 2005.

4. Bertoldo F, Santini D and Lo Cascio V: Bisphosphonates and osteomyelitis of the jaw: A pathogenic puzzle. Nat Clin Pract Oncol 4: 711-721, 2007.

5. Shipman CM, Rogers MJ, Apperley JF, Russell RG and Croucher PI: Bisphosphonates induce apoptosis in human myeloma cell lines: A novel anti-tumour activity. Br J Haematol 98: 665-672, 1997.

6. Van Beek ER, Cohen LH, Leroy IM, Ebetino FH, Löwik CW and Papapoulos SE: Differentiating the mechanisms of antiresorptive action of nitrogen containing bisphosphonates. Bone 33: 805-811, 2003.

7. Rogers MJ, Crockett JC, Coxon FP and Mönkkönen J: Biochemical and molecular mechanisms of action of bisphosphonates. Bone 49: 34-41, 2011.

8. Hughes DE, MacDonald BR, Russell RG and Gowen M: Inhibition of osteoclast-like cell formation by bisphosphonates in long-term cultures of human bone marrow. J Clin Invest 83: 1930-1935, 1989.

9. Löwik CW, van der Pluijm G, van der Wee-Pals LJ, van Treslong-De Groot HB and Bijvoet OL: Migration and phenotypic transformation of osteoclast precursors into mature osteoclasts: The effect of abisphosphonate. J Bone Miner Res 3: 185-192, 1988.

10. Fleisch H, Russell RG and Francis MD: Diphosphonates inhibit hydroxyapatite dissolution in vitro and bone resorption in tissue culture and in vivo. Science 165: 1262-1264, 1969.

11. Coxon FP, Helfrich MH, Van't Hof R, Sebti S, Ralston SH, Hamilton A and Rogers MJ: Protein geranylgeranylation is required for osteoclast formation, function and survival: Inhibition by bisphosphonates and GGTI-298. J Bone Miner Res 15: 1467-1476, 2000.
12. Marx RE: Pamidronate (Aredia) and zoledronate (Zometa) induced avascular necrosis of the jaws: A growing epidemic. J Oral Maxillofac Surg 61: 1115-1117, 2003.

13. Costa L, Lipton A and Coleman RE: Role of bisphosphonates for the management of skeletal complications and bone pain from skeletal metastases. Support Cancer Ther 3: 143-153, 2006.

14. Mawardi H, Giro G, Kajiya M, Ohta K, Almazrooa S, Alshwaimi E, Woo SB, Nishimura I and Kawai T: A role of oral bacteria in bisphosphonate-induced osteonecrosis of the jaw. J Dent Res 90: 1339-1345, 2011.

15. Fournier P, Boissier S, Filleur S, Guglielmi J, Cabon F, Colombel $\mathrm{M}$ and Clézardin P: Bisphosphonates inhibit angiogenesis in vitro and testosterone-stimulated vascular regrowth in the ventral prostate in castrated rats. Cancer Res 62: 6538-6544, 2002.

16. Kobayashi Y, Hiraga T, Ueda A, Wang L, Matsumoto-Nakano M, Hata K, Yatani $\mathrm{H}$ and Yoneda T: Zoledronic acid delays wound healing of the tooth extraction socket, inhibits oral epithelial cell migration and promotes proliferation and adhesion to hydroxyapatite of oral bacteria, without causing osteonecrosis of the jaw, in mice. J Bone Miner Metab 28: 165-175, 2010.

17. Khokher MA and Dandona P: Diphosphonates inhibit human osteoblast secretion and proliferation. Metabolism 38: 184-187, 1989.

18. De Ponte FS, Favaloro A, Siniscalchi EN, Centofanti A, Runci M, Cutroneo G and Catalfamo L: Sarcoglycans and integrins in bisphosphonate treatment: Immunohistochemical and scanning electron microscopy study. Oncol Rep 30: 2639-2646, 2013.

19. Nastro Siniscalchi E, Cutroneo G, Catalfamo L, Santoro G, Allegra A, Oteri G, Cicciù D, Alonci A, Penna G, Musolino C, et al: Immunohistochemial evaluation of sarcoglycans and integrins in gingival epithelium of multiple myeloma patients with bisphosphonate-induced osteonecrosis of the jaw. Oncol Rep 24: 129-134, 2010.

20. Senel FC, Kadioglu Duman M, Muci E, Cankaya M, Pampu AA, Ersoz S and Gunhan O: Jaw bone changes in rats after treatment with zoledronate and pamidronate. Oral Surg Oral Med Oral Pathol Oral Radiol Endod 109: 385-391, 2010

21. Su J, Feng M, Han W and Zhao H: The effects of bisphosphonate on the remodeling of different irregular bones in mice. J Oral Pathol Med 44: 638-648, 2015.

22. Pautke C, Kreutzer K, Weitz J, Knödler M, Münzel D, Wexel G, Otto S, Hapfelmeier A, Stürzenbaum S and Tischer T: Bisphosphonate related osteonecrosis of the jaw: A minipig large animal model. Bone 51: 592-599, 2012.

23. Pytlik M, Kaczmarczyk-Sedlak I, Sliwiński L, Janiec W and Rymkiewicz I: Effect of concurrent administration of alendronate sodium and retinol on development of changes in histomorphometric parameters of bones induced by ovariectomy in rats. Pol J Pharmacol 56: 571-579, 2004.

24. Kapitola J, Zák J, Lacinová Z and Justová V: Effect of growth hormone and pamidronate on bone blood flow, bone mineral and IGF-I levels in the rat. Physiol Res 49 (Suppl 1): S101-S106, 2000.

25. O'Ryan FS and Lo JC: Bisphosphonate-related osteonecrosis of the jaw in patients with oral bisphosphonate exposure: Clinical course and outcomes. J Oral Maxillofac Surg 70: 1844-1853, 2012.

26. Ruggiero SL, Carlson ER and Assael LA: Comprehensive review of bisphosphonate therapy: Implications for the oral and maxillofacial surgery patient. J Oral Maxillofac Surg 67 (Suppl 5): S1, 2009.

27. Arco A, Favaloro A, Gioffrè M, Santoro G, Speciale F, Vermiglio G and Cutroneo G: Sarcoglycans in the normal and pathological breast tissue of humans: An immunohistochemical and molecular study. Cells Tissues Organs 195: 550-562, 2012.

28. Hynes RO: Integrins: Versatility, modulation and signaling in cell adhesion. Cell 69: 11-25, 1992

29. Trimarchi F, Favaloro A, Fulle S, Magaudda L, Puglielli C and Di Mauro D: Culture of human skeletal muscle myoblasts: Timing appearance and localization of dystrophin-glycoprotein complex and vinculin-talin-integrin complex. Cells Tissues Organs 183: 87-98, 2006.

30. Cankaya AB,Erdem MA, Isler SC, Demircan S, Soluk M,Kasapoglu $\mathrm{C}$ and Oral CK: Use of Cone-Beam Computerized Tomography for Evaluation of Bisphosphonate-Associated Osteonecrosis of the Jaws in an Experimental Rat Model. Int J Med Sci 8: 667-672, 2011.

31. Ali-Erdem M, Burak-Cankaya A, Cemil-Isler S, Demircan S, Soluk M, Kasapoglu C and Korhan-Oral C: Extraction socket healing in rats treated with bisphosphonate: Animal model for bisphosphonate related osteonecrosis of jaws in multiple myeloma patients. Med Oral Patol Oral Cir Bucal 16: 879-883, 2011. 
32. Murakami H, Takahashi N, Sasaki T, Udagawa N, Tanaka S, Nakamura I, Zhang D, Barbier A and Suda T: A possible mechanism of the specific action of bisphosphonates on osteoclasts: Tiludronate preferentially affects polarized osteoclasts having ruffled borders. Bone 17: 137-144, 1995.

33. Biver E, Vieillard MH, Cortet B, Salleron J, Falgayrac G and Penel G: No anti-angiogenic effect of clinical dosing regimens of a single zoledronic acid injection in an experimental bone healing site. Bone 46: 643-648, 2010.

34. Tanaka Y, Nagai Y, Dohdoh M, Oizumi T, Ohki A, Kuroishi T, Sugawara $\mathrm{S}$ and Endo $\mathrm{Y}$ : In vitro cytotoxicity of zoledronate (nitrogen-containing bisphosphonate: NBP) and/or etidronate (non-NBP) in tumour cells and periodontal cells. Arch Oral Biol 58: 628-637, 2013.

35. Lustosa-Pereira A, Garcia RB, de Moraes IG, Bernardineli N, Bramante CM and Bortoluzzi EA: Evaluation of the topical effect of alendronate on the root surface of extracted and replanted teeth. Microscopic analysis on rats' teeth. Dent Traumatol 22: 30-35, 2006
36. Lekic P, Rubbino I, Krasnoshtein F, Cheifetz S, McCulloch CA and Tenenbaum $\mathrm{H}$ : Bisphosphonate modulates proliferation and differentiation of rat periodontal ligament cells during wound healing. Anat Rec 247: 329-340, 1997.

37. Yoshida M and Ozawa E: Glycoprotein complex anchoring dystrophin to sarcolemma. J Biochem 108: 748-752, 1990.

38. Ervasti JM and Campbell KP: A role for the dystrophin-glycoprotein complex as a transmembrane linker between laminin and actin. J Cell Biol 122: 209-231, 1993.

39. Campbell KP: Three muscular dystrophies: Loss of cytoskeleton-extracellular matrix linkage. Cell 80: 675-679, 1995.

40. American Association of Oral and Maxillofacial Surgeons (AAOMS): Office Anesthesia Evaluation Manual. 8th edition. AAOMS, Rosemont, IL, USA, pp1899-1900, 2011. 